\title{
Transient Tachypnea of the Newborn and the Use of Prophylactic Antibiotics
}

\author{
Dalal Saadoun*, Leen Doya, Adnan Dayoub, Oday Jouni \\ Pediatrics Department, Tishreen Hospital, Faculty of Medicine, Tishreen University, Lattakia, Syria \\ Email address: \\ dr.dalal.saadoun@gmail.com (D. Saadoun),dr.leen.doya@gmail.com (L. Doya), adnan.1957.dayoub@gmail.com (A. Dayoub), \\ odayjouni22@gmail.com (O. Jouni) \\ ${ }^{*}$ Corresponding author
}

To cite this article:

Dalal Saadoun, Leen Doya, Adnan Dayoub, Oday Jouni. Transient Tachypnea of the Newborn and the Use of Prophylactic Antibiotics. Journal of Drug Design and Medicinal Chemistry. Vol. 7, No. 1, 2021, pp. 1-4. doi: 10.11648/j.jddmc.20210701.11

Received: July 29, 2020; Accepted: August 10, 2020; Published: January 22, 2021

\begin{abstract}
Background: Transient tachypnea of the newborn (TTNB) is a common cause of respiratory distress in late preterm and full-term infants. It is caused by a delay in the clearance of fetal lung fluids. Although TTNB is a self-limited condition, prophylactic antibiotics usually administered for 48-72 hours until negative blood culture is reported. Objective: In this study, we aim to identify the relationship between using prophylactic antibiotics and the duration of tachypnea and hospitalization in neonates with TTNB. Materials and Methods: this was a cohort study design included 102 infants with TTNB. The infants were divided into two groups, one received supportive care, and the other received supportive care with intravenous antibiotics. The clinical signs and laboratory results were examined in the two groups. Results: Of total 102 infants who were included in this study, $41(40.2 \%)$ were received supportive care with prophylactic intravenous antibiotics. There were no significant differences between two groups in terms of gender, gestational age, birth weight, mode of delivery, and white blood cell. A significant relation was found between receiving prophylactic antibiotics with the duration of tachypnea and hospitalization. Conclusions: In the recent study, we found an increase in the hospitalization and tachypnea period in the group received antibiotics.
\end{abstract}

Keywords: Transient Tachypnea of the Newborn, Prophylactic Antibiotics, Duration of Tachypnea, Duration of Hospitalization

\section{Introduction}

Transient Tachypnea of the Newborn (TTNB) is the most commonly diagnosed respiratory condition in late preterm and term newborn infant, characterized by pulmonary edema resulting from delayed reabsorption and clearance of the fetal alveolar fluid [1]. Several risk factors for TTNB have been identified in the literature included cesarean delivery, male gender, small for gestational age, maternal asthma and diabetes, and breech delivery [2]. The incidence of TTNB varies from 4 to 11 cases per 1000 live birth [3]. The onset of TTNB is usually at the time of birth and within 6 hours after delivery. Tachypnea (respiratory rate greater than 60 breaths per minute) is the most prominent feature. Infants with this condition typically have increased work of breathing, manifested by nasal flaring, mild intercostal and subcostal retractions, and expiratory grunting [4]. TTNB is a benign self-limited condition that usually resolves within 48-72hours [5], and requires only monitoring and supportive treatment in neonatal intensive care (NICU) [6]. However some doctors use intravenous antibiotics for treatment, based on a terror of hidden infections [7], and because the clinical symptoms are not specific [8]. Growing awareness of the increase of antibiotic resistance, side effects, and other potential risks of antibiotics prompting clinicians to find ways to minimize their unnecessary use in neonates. We aim to compare the duration of tachypnea and hospitalization in two groups of infants with TTNB, one treated and the other not treated with prophylactic antibiotics. 


\section{Material and Methods}

A non-randomized prospective cohort study included 102 infants with TTNB admitted to NICU in Tishreen University Hospital in Latakia during one year, from April 2019 to April 2020.

Inclusion criteria included infants whose gestation ages between 37- 41 weeks with a diagnosis of TTNB. Criteria used to confirm the diagnosis of TTNB were as follow: clinical signs of dyspnea (including tachypnea rate up to 60 breath/minute, grunting, retractions and respiratory distress) beginning during the first 6 hours of birth with a chest-X-ray demonstrating fluid retention and absent of any competing diagnosis to explain the respiratory distress. Infants were excluded from the study if there were any of the following exclusion criteria:(1) antenatal history of (Prelab or rupture of membranes (PROM) > 18hours, (2) maternal infection, chorioamnionitis, (3) meconium aspiration, (4) congenital malformations, (5) the neonate was diagnosed with a bacterial infection, such as bacterial pneumonia, bacterial septicemia, or bacterial meningitis immediately following admission to the hospital.

Data was collected by recording the following information: demographic information (sex of neonate, gestational age, birth weight, mode of delivery, pregnancy and childbirth history and the presence of risk factors for infection).

Lab tests were performed for all infants at the admission and at the age of 24 hours including blood culture, complete blood cell (CBC), C-reactive protein (CRP). Chest-X-ray were performed at the admission. Infants divided into two groups according to the opinion of the Dr. supervisor, one received supportive care (61infants), and the other received supportive care with prophylactic intravenous antibiotics (41infants). We followed them up during the TTNB duration and hospitalization.

All data were analyzed using the Statistical Package for Social Sciences (SPSS Version (19). Descriptive data are reported as mean \pm standard deviation or number (percentage) as appropriate. Chi-Square test and Independent $\mathrm{T}$ student were used for comparison of categorical variables. Results were considered statistically significant with a $\mathrm{p}$-value $<5 \%$.

\section{Results}

In total 102 neonates with transient tachypnea 63 $(61.80 \%)$ were males and $39(38.20 \%)$ were females. Of all neonates $97.1 \%$ was born in caesarean section. The gestational ages ranged between (37-39) gestational week with the mean $(37.6 \pm 0.7)$ week. The birth weights ranged between (1900-3950) grams with the mean (2832.8 \pm 436.8$)$ grams. The percentage of neonates who received prophylactic antibiotics was $40.2 \%$ of all neonates. There was no significant difference in gestational age, birth weight, gender ratio, mode of delivery, and the mean of white blood cells (Table 1).

Table 1. Comparison between neonates with and without using antibiotic.

\begin{tabular}{|c|c|c|c|}
\hline Variables & With antibiotic & Without antibiotic & P-value \\
\hline \multicolumn{4}{|l|}{ Gender } \\
\hline Male (\%) & $25(61 \%)$ & $38(62.3 \%)$ & 0.8 \\
\hline Female $(\%)$ & $16(39 \%)$ & $23(37.7 \%)$ & \\
\hline Caesarean delivery & $40(97.6 \%)$ & $59(96.7 \%)$ & 0.6 \\
\hline vaginal birth & $1(2.4 \%)$ & $2(3.3 \%)$ & \\
\hline Gestation age (weeks) & $37.4 \pm 0.5$ & $37.7 \pm 0.8$ & 0.1 \\
\hline $\mathrm{WBC}\left(\right.$ cell / mm $\left.{ }^{3}\right)$ & $14.2 \pm 3.7$ & $13.9 \pm 3.1$ & 0.6 \\
\hline
\end{tabular}

WBC: white blood cells

The recent study found a significant difference between the two groups for hospitalization period ( $p$-value $=0.0001$ ), with an increase in the duration of hospitalization for the group that received prophylactic antibiotics (Table 2).

Table 2. Comparison between neonates with and without using antibiotic according to Duration of hospitalization.

\begin{tabular}{lll}
\hline Duration of hospitalization & With antibiotics & Without antibiotics \\
\hline$\leq 3$ Days & 0 & $40(65.6 \%)$ \\
[3-7] days & $13(31.7 \%)$ & $19(31.1 \%)$ \\
$>$ 7days & $28(68.3 \%)$ & $2(3.3 \%)$ \\
Total & 41 & 61 \\
\hline
\end{tabular}

There was a significant difference between the two groups in terms of the duration of tachypnea ( $\mathrm{p}$-value $=0.002$ ), with a longest period in the group that received antibiotics (Table 3).

Table 3. Comparison between neonates with and without using antibiotic according to Duration of tachypnea.

\begin{tabular}{lll}
\hline Duration of tachypnea & With antibiotics & Without antibiotics \\
\hline$\leq 72$ hours & $26(63.4 \%)$ & $55(90.2 \%)$ \\
$>72$ hours & $15(36.6 \%)$ & $6(9.8 \%)$ \\
Total & 41 & 61 \\
\hline
\end{tabular}




\section{Discussion}

The use of antibiotics in newborns even for only a short period appears to be associated with multiple health risks [9]. Antibiotic causes alterations in gut microflora, which can predispose them to several complications [10], increase the prevalence of antibiotic resistance, nephrotoxic and ototoxic complications. Thus, the prescription of these drugs in the absence of specific risk factors of infection should be limited to the necessary cases $[11,12]$.

Although more and more infection indicators are being used in clinical practice, bacterial culture remains the gold standard for diagnosing bacterial infection [13]. In the current study, there were no pathognomonic, clinical presentations of neonatal sepsis or signs of septicemia, in neonates. There were no significant differences between complete white blood cell count, CRP, and blood culture in the two groups. In our study, the duration of tachypnea was 72 hours or less in $90.2 \%$ of neonates received supportive care, whereas it was 72 hours or less in $63 \%$ of neonates received supportive care and prophylactic antibiotics. This result has not been studied yet by any other study. In the current study the duration of hospitalization was longer in group treated with antibiotics compared to the untreated group that was similar to Sabzehei et al 2018 in Iran who found that infants with TTNB not treated with antibiotics have a shorter length of hospitalization [14]. Yarci et al in Turkey 2019 found that infants with TTNB who not treated with antibiotics have a shorter length of hospitalization. Consequently, they are not exposed to complications related to long stay in hospital [15]. The current study did not find any complication in the infants in two groups. Dehdashtian et al study has also shown that empirical use of antibiotics may not be necessary for infants with TTNB, and prolonged hospitalization while awaiting blood culture results may be unwarranted and is associated with high bed occupancy rates and high economic costs [16]. In contrast, Weintraub et al reported no difference in median length of hospital stay between the treated and untreated groups. Similarly, in 2015, Li et al reported no difference between the control and treated groups [17, 18].

This study has several limitations, as this is a single-center study and the decision for prophylactic intravenous antibiotics is usually taken by the attending physician. Second, the total number of cases examined was not sufficient to generalize the conclusion. Third, we could not demonstrate long-term serious complications and outcomes.

\section{Conclusion}

This study was the first study in Syria showed that adding the Prophylactic antibiotics as part of the treatment of classic TTNB protocols was useless. We recommended avoiding the use of unnecessary Prophylactic antibiotics in newborns with the diagnosis of TTNB, with careful monitoring for any clinical sign of sepsis.

\section{List of Abbreviations}

TTNB: Transient Tachypnea of the Newborn.

NICU: neonatal intensive care.

PROM: Pre labor rupture of membranes.

CBC: complete blood cell.

CRP: C-reactive protein.

\section{Statement of Ethics}

All parents whose children were studied gave informed consent for the sharing of this research. Ethical clearance for this study was obtained from the Ethical Committee of the University of Tishreen Hospital.

\section{Funding Sources}

None.

\section{Conflict of Interest Statement}

The authors declare that they have no competing interests.

\section{Author Contribution}

Both authors developed and carried out sample collection. A Literature review was done by Dr. Dalal Saadoun, and both authors did the data analysis and read through the final data.

\section{Acknowledgements}

We wish to thank the medical officer, doctors in the neonatal department, and laboratory assistants in Tishreen Hospital laboratory who worked with us in reviewing the patients and doing the laboratory analysis. We want to thank miss Alesar Hejazia and Dr. Asmaa Saadoun for their technical help.

\section{References}

[1] Avery, M. E; Gatewood, O. B; Brumley, G. Transient tachypnea of newborn. Possible delayed resorption of fluid at birth. Am J Dis Child 1966; 111: 380.

[2] Hamdoon, G. W. Risk factors for development of transient tachypnea of newborns. Annals of the College of Medicine Mosul 2018; 40, no. 1: 15-19.

[3] Morrison, J. J; Rennie, J. M; Milton, P. J. Neonatal respiratory morbidity and mode of delivery at term: influence of timing of elective caesarean section. Br J Obstet Gynaecol 1995; 102: 101.

[4] Aathi, M. K. "Transient Tachypnea of Newborn (TTN): An Overview." Int J Nur Edu Res 2014; 2, no. 2: 99-103.

[5] Morioka, I; Yamana, K; Kurokawa, D. How long is transient tachypnea of the newborn dependent on oxygen supplementation? Pediatr Int 2015; 57: 1054-1055. 
[6] Hagen, E; Chu, A; Lew, Ch. Transient Tachypnea of the Newborn. American Academy of Pediatrics 2017; 18 (3): 141148 .

[7] Abughalwa, M; Taha, S; Sharaf, N; Salama, H. Antibiotics therapy in classic transient tachypnea of the newborn: A necessary treatment or not? A prospective study. Neonatology Today $2012 ; 7$.

[8] Leibovici, L; Paul, M; Garner, P; Sinclair, D. J; Afshari, A; Pace, N. L. Addressing resistance to antibiotics in systematic reviews of antibiotic interventions. The Journal of Antimicrobial Chemotherapy 2016; 71 (9): 2367-9.

[9] Bromberger, P; Lawrence, J. M; Braun, D; Saunders, B; Contreras, R; Pettiti, D. B. The influence of intrapartum antibiotics on the clinical spectrum of early-onset group B streptococcal infection in term infants. Pediatrics 2000; 106: 244-50.

[10] Zhang, S; Chan, D. Facing a new challenge; the adverse effects of antibiotics on gut microbiota and host immunity. Chin Med J 2019; 132 (10) 1135-1138.

[11] Cipolla, D; Giuffrè, M; Mammina, C; Corsello, G. Prevention of nosocomial infections and surveillance of emerging resistances in NICU. The Journal of Maternal-Fetal \& Neonatal Medicine 2011; 24 (sup1): 23-6.

[12] Yurdakok, M; Ozek, E. Transient tachypnea of the newborn: the treatment strategies. Current pharmaceutical design 2012; 18 (21): 3046-9.
[13] Tripathi, N; Cotten, C. M; Smith, P. B. Antibiotic use and misuse in the neonatal intensive care unit. Clin Perinatol 2012; 39 (1): 61-68. doi: 10.1016/j.clp.2011.12.003.

[14] Sabzehei, M. K; Basiri, B; Shokouhi, M; Poorolajal, J; \& Bahadorbeigi, L. Evaluating the Need for Prophylactic Antibiotic Therapy in Infants with Transient Tachypnea of the Newborn: A Triple-Blind Randomized Clinical Trial Study. International Journal of Pediatrics2018; 6 (8), 8086-8092.

[15] Yarci, E; Asihan, K. C; Fuat, E. C. "Antibiotics for transient tachypnea of newborn; are comorbidities reasons or results?" Jinekoloji-Obstetrik ve Neonatoloji Tip Dergisi 2019; 17, no. 1: 254-257.

[16] Dehdashtian, M; Aletayeb, M; Malakian, A; Aramesh, M. R, \& Malvandi, H. Clinical course in infants diagnosed with transient tachypnea of newborn: A clinical trial assessing the role of conservative versus conventional management. Journal of the Chinese Medical Association 2018; 81 (2), 183-186.

[17] Weintraub, A. S; Cadet, C. T; Perez, R; DeLorenzo, E; Holzman, I. R; Stroustrup, A. Antibiotic use in newborns with transient tachypnea of the newborn. Neonatology 2013; 103 (3): 235-240. doi: 10. 1159/000346057.

[18] Li, J; Wu, J; Du, L; Hu, Y; Yang, X; Mu, D; Xia, B. Different antibiotic strategies in transient tachypnea of the newborn: an ambispective cohort study. Eur J Pediatr 2015; 174 (9): 1217 23. 